proportion of cases were extremely complex. Nevertheless we were interested to find that among the tumours in the diploid range $(20 / 52,38 \%)$ there were some which could be investigated in detail and a few with only little chromosome change.

Even more interesting was the finding that of the three tumours which carried the least chromosome change the simplest was pseudodiploid, having only monosomy 16 and the marker del (1)(qter->p21:) and identical changes were seen in a second tumour although there was an additional marker in this case. The third tumour was slightly more complex, and included in the seven markers were a chromosome 1 marker $\mathrm{t}(1 ; 2)$ (1qter->1q21::2q33->2qter) and also a deleted chromosome 16 , $\operatorname{del}(16)(q t e r->$ p21:).

It seems reasonable to conclude that any search for somatic gene mutations in breast carcinoma should be directed to either chromosome 16 or to the long arm of chromosome 1. As markers involving the long arm of chromosome 1 have also been shown for other carcinomas and malignancies such as myelomas, lymphomas and melanomas, it seems that monosomy and thus hemizygosity for chromosome 16 might be the more relevant.

Maj Hulten

Regional Cytogenetics Laboratory,

East Birmingham Hospital,'

Bordesley Green East,

Birmingham B9 5ST, UK

\section{Mutualism disagreement}

SIR - We have commented previously on May's views about the global distribution of mutualism ${ }^{1,2}$. In his recent article ${ }^{3}$, he contrasts "tight mutualistic associations between clearly distinct species" and "entities like lichens, where algae and fungi are fused into something akin to a single organism'. He then excludes the latter types from his considerations. This evades the fact that the participants of lichens and similar associations (Table 1 of ref. 4 lists 25 such kinds) still have separate identities and genetics, and distinct (yet interesting) population dynamics and patterns of evolution. Sounder reasons for selection of particular sub-sets from the range of mutualisms available are required.

The supposed (but disputed ${ }^{2}$ ) inconspicuousness of ecologically obligate mutualisms in temperate regions is used to provide backing to a theory, also mentioned by $\mathrm{May}^{3}$, which predicts that such mutualisms are dynamically fragile and therefore more likely to be found in tropical environments, assumed to be more stable than temperate ones. Clearly, if this theory is tested on data from which obligate mutualisms common in temperate environments have been excluded, the conclusions reached are unlikely to reflect reality. It would be more instructive to assess those features of the theory which are responsible for such an unrealistic prediction about the geographical distribution of obligate mutualists. One likely feature is the theory's lack of a spatial dimension, for it can be demonstrated that obligate mutualists are more resilient against environmental changes if they are able to diffuse through space ${ }^{5,6}$.

\section{Department of Biology, \\ University of York, York YOI SDD, UK}

\section{Department of Applied Mathematics,} University of Sheffield

\section{Department of Botany}

University of Sheffield, Sheffield S10 2TN, UK

1. May, R.M. Nature 296, 803 (1982).

2. Lewis, D.H. Nature 297,176 (1982)

3. May, R.M. Nature 307,410 (1984)

4. Law, R. \& Lewis, D.H. Biol. J. Linn. Soc. 20, 249 (1983).

5. Hutson, V. SIAM J. appl. Math. (submitted)

6. Hutson, V., Law, R \& Lewis, D. Am Nat. (submitted)

\section{Archaeopteryx's morphology}

SIR - Hecht and Tarsitano ${ }^{1}$, in their response to my necessarily brief News and Views article", accuse me of "distorting and misrepresenting (their) viewpoints" concerning the morphology and homologies of the manus of Archaeopteryx. In my article I was concerned solely to highlight aspects of disputed morphology which could be settled by a comparison of the actual specimens, if they could be gathered together for the forthcoming Eichstatt symposium.

Nowhere in the article did I refer to the homologies of the digits of Archaeopteryx, either with modern birds or theropod dinosaurs; nor did I suggest that Archaeopteryx might have a digital reduction pattern of 1.2.3 as Hecht and Tarsitano state' ${ }^{\perp}$ I can only assume that because I favoured a phalangeal formula of 2.3.4, Hecht and Tarsitano presumed that I must also favour the preservation of the ancestral phalangeal formula and hence a 1.2.3 digital reduction pattern. However, a phalangeal formula of 2.3.4 can be derived via a 1.2.3 or 2.3.4 digital reduction pattern from a primitive reptilian manus of five digits with a phalangeal formula of 2.3 .4 .5 .3 . If the 2.3.4 digital reduction pattern is accepted, then a 2.3.4 phalangeal formula is less derived than would be the case if the broken phalanx hypothesis is accepted, as this would give a phalangeal formula of 2.3.3.

On the question of this supposed break in the topographic third digit, Hecht and Tarsitano are quite definite. "We regard the articulation between the first and second phalanges as displacement and breakage" $^{3}$ (p.161), and their illustrations (pp.156-157) label the joint as a "break within (the) first phalanx of the fourth digit". After criticism of this point ${ }^{4}$ they become more circumspect, but still argue the case for a break ${ }^{5}$, citing Heller's ${ }^{6}$ mistaken restoration (see plate 7 of ref.6) of the left manus of the Maxberg specimen as an example of an unbroken phalanx. However, a perusal of the accompanying photographs (plates 9 and 11 of ref.6)

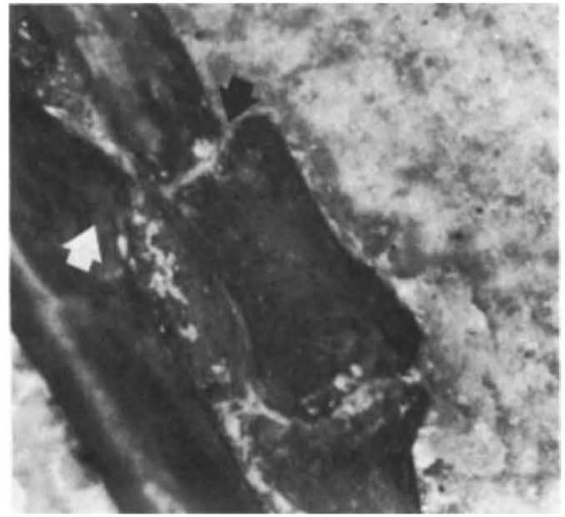

Fig.1 The left manus of the Berlin specimen showing the supposed break (actually a joint, black arrow) in the first phalanx of the third topographic digit and the outgrowth (the "flange" of Hecht and Tarsitano, white arrow) on the first phalanx of the second topographic digit.

reveals the "unbroken phalanx" as two distinct impressions, and my own detailed photographs of the critical area of the Berlin specimen ${ }^{2}$ (Fig. 1) show that the supposed break is as good a joint as the other, undisputed joints illustrated.

My second "distortion and misinterpretation" concerns the function of the supposed flange. Hecht and Tarsitano ${ }^{3}$ regard this structure as "a brace or point of attachment" (p.162). In the absence of any explanation as to what this means I think that I am entitled to describe the function as "to brace and strengthen" 2 . I might add that to brace a part of the wing serves to brace the structure as a whole.

The flange, however, appears to be a pathological outgrowth as it has a different texture from the smooth bone of the phalanx, being more rugose. It also has a distinct margin, which cuts across (overgrows?) a linear structure near the left-hand margin of the exposed bone (see Fig.1).

As for Hecht and Tarsitano's "crashdive" scenario", a joint is not "caused by forces during stalling (or) ... impact"!, only a break. The crossing of the fingers, which can be seen in the Berlin and Eichstatt specimens (both hands) and the Maxberg specimen (one hand), are attributed to the same "catastrophic" cause. This twisting has a much more parsimonious explanation as being due to the heeling over of the strongly arched claws sideways ${ }^{4}$ as the dead animal settled on the sea bed. Hecht and Tarsitano do their case for the homology of avian digits with those of $\mathrm{Ar}$ chaeopteryx no good by a myopic view of palaeontology and an extravagant use of $a d$ hoc scenarios.

Department of Zoology, M.E. HowgATE

University College London, Gower Street, London WCI 6BT, UK

1. Hecht, M.K. \& Tarsitano, S. Nature 309, 588 (1984)

2. Howgate, M.E. Nature 306, 644 (1983)

. Tarsitano, S. \& Hechx, M.K. J. Linn. Soc. Zool. 69, 149 (1979).

4. Thulborn, R.A. \& Hamley, T.L. Aust. J. Zool. 30, 611 (1982).

5. Hecht. M.K. \& Tarsitano, S. S. Geogios mem. spec. 6, 141 (1982)

6. Heller, F. Erlanger Geol. Abht. 31, 3 (1959) 